\title{
The neural correlates of the body-object interaction effect in semantic processing
}

\author{
lan S. Hargreaves ${ }^{1}{ }^{*}$, Gemma A. Leonard ${ }^{1}$, Penny M. Pexman ${ }^{1}$, Daniel J. Pittman ${ }^{3}$, Paul D. Siakaluk ${ }^{4}$ \\ and Bradley G. Goodyear ${ }^{2,3}$ \\ 1 Department of Psychology, University of Calgary, Calgary, AB, Canada \\ 2 Department of Radiology, University of Calgary, Calgary, AB, Canada \\ ${ }^{3}$ Seaman Family MR Research Centre, University of Calgary, Calgary, AB, Canada \\ ${ }^{4}$ Department of Psychology, University of Northern British Columbia, Prince George, Canada
}

\section{Edited by:}

Melvin Yap, National University of

Singapore, Singapore

Reviewed by:

Carrie Esopenko, Rotman Research Institute, Canada

Anna M. Woollams, University of Manchester, UK

*Correspondence:

lan S. Hargreaves, Department of Psychology, University of Calgary,

2500 University Drive NW, Calgary, AB T2N 1N4, Canada.

e-mail: ishargre@ucalgary.ca
The semantic richness dimension referred to as body-object interaction (BOI) measures perceptions of the ease with which people can physically interact with words' referents. Previous studies have shown facilitated lexical and semantic processing for words rated high in BOI, e.g., belt, than for words rated low in BOI, e.g., sun. These BOI effects have been taken as evidence that embodied information is relevant to word recognition (Siakaluk et al., 2008a). However, to date there is no evidence linking BOI manipulations to differences in the utilization of perceptual or sensorimotor areas of the brain. The current study used event-related functional magnetic resonance imaging (fMRI) to examine the neural correlates of BOI in a semantic categorization task (SCT). Sixteen healthy adults participated. Results showed that high BOI words were associated with activation in the left inferior parietal lobule (supramarginal gyrus, BA 40), a sensory association area involved in kinesthetic memory. These results provide evidence that the $\mathrm{BOI}$ dimension captures the relative availability of sensorimotor information, and that this contributes to semantic processing.

Keywords: semantic richness, body-object interaction, semantic processing, semantic categorization task, fMRI, sensorimotor
Our ability to efficiently extract information from the world around us is a crucial component of human cognition. The debate concerning how and what information is preserved in memory has been a topic of interest to cognitive scientists since the latter half of the twentieth century. Heavily influenced by functionalism, much of this work has focused on the explanatory power of computational models (Pylyshyn, 1984). This yielded a literature with numerous models of conceptual representation, each providing a specification of the mechanisms by which humans acquire and represent information (Morton, 1969; Collins and Loftus, 1975). Though these models often differed in architectural principles, many authors agreed (at least tacitly) that conceptual representation should be understood as fundamentally amodal and symbolic in nature (Pylyshyn, 1984). Recent work has expanded upon this framework, arguing that a more refined model of semantic processing requires the contributions of both amodal systems and modality-specific systems (Patterson et al., 2007; Dove, 2009). An alternative approach known as embodied cognition goes further still, arguing that most, if not all, of semantic processing is tied to the sensory and motor systems that guide our interactions with the world (Barsalou, 1999; Gibbs, 2006; Pulvermüller, 2010).

Since our histories of sensorimotor interactions help to form conceptual representations, many theories of embodied cognition predict the existence of sensorimotor effects during all kinds of processing. This is the case even for processing that is thought to be of a very abstract character, such as the processing of written language. Wilson (2002) argued that the same mechanisms that are involved in perception and action should also play a role in cognition that is decoupled from the processing of the immediate environment. That is, during "off-line" processing where the immediate environment is merely referenced (e.g., when reading individual words) modality-specific systems will still be recruited in order to "assist in thinking and knowing" (p. 633). These predictions have inspired investigations of sensorimotor effects in basic lexical processes.

Using auditorily presented words in a lexical decision task (LDT: "Is it a real English word?"), Myung et al. (2006) found that participants were faster to verify a target word (e.g., "baseball") when it was preceded by a prime that shared manipulation features (e.g., "grenade") than when compared to an unrelated prime (e.g., "leaflet"). The Myung et al. results suggest that the sensorimotor information in the meanings of auditorialy presented nouns is automatically recruited during a LDT, and that overlap in sensorimotor information between meanings can facilitate word recognition. Using a LDT and a phonological lexical decision task (PLDT: "Does it sound like a real English word?") with visually presented words, Siakaluk and colleagues found significant facilitation for words that were rated high in body-object interaction (BOI), a dimension which measures perceptions of the ease with which a human body can physically interact with a word's referent (Siakaluk et al., 2008a; Tillotson et al., 2008). These effects were observed for a set of high BOI items that were matched with a set of low BOI items on numerous lexical 
and semantic dimensions such as imageability and concreteness. Thus, the incremental effects of BOI provide evidence that lexical semantics includes information about sensorimotor experiences, and that the relative availability of this information can influence word recognition (Pexman et al., 2002). Using a semantic categorization task (SCT: “is the words' referent easily imageable?”) that is thought to focus more on the activation of meaning per se than either the LDT or PLDT, Siakaluk and colleagues observed significant facilitation for words that were rated high on the BOI dimension; that is, faster and more accurate categorization for high BOI words than for low BOI words (Siakaluk et al., 2008b; Bennett et al., 2011; Wellsby et al., 2011). Again, the authors interpreted these significant BOI effects as evidence that sensorimotor information is incorporated in lexical semantics and that this information influences off-line cognition in which the physical environment is merely referenced using language.

Along with BOI effects, other research has demonstrated that participants' metalinguistic judgments about the sensorimotor characteristics of words are able to predict word recognition performance. In a related study by Juhasz and colleagues (Juhasz et al., 2011) participants were directed to not only consider the ease of embodied interaction with the referent of a given word's meaning, but also the degree to which additional sensory experiences (e.g., taste, smell, sight, sound) are evoked by that word. These sensory experience ratings (SERs) were found to account for a significant and unique proportion of variance in LDT reaction times for over 2000 words from the British Lexicon Project (Keuleers et al., 2012). While BOI ratings are assumed to capture sensorimotor experience, the additional variance in LDT that SER ratings accounts for is thought to result from the added contributions of the other senses. However, this explanation relies on an untested assumption that is the focus of the present research. Though Siakaluk and colleagues have consistently found evidence of BOI effects in lexical semantic tasks, questions remain as to what BOI ratings actually capture. Possibilities include proprioceptive or kinesthetic information, such as motor programs for effectively interacting with the environment that are then stored in memory (Gibbs, 2006). However, it is possible that many kinds of information influence participants' BOI ratings, and to date there is no evidence linking BOI effects to activation in areas of the brain dedicated to sensorimotor or kinesthetic memory. The objective of the current study is to test the assumption that BOI ratings capture sensorimotor experience by investigating the neurophysiological correlates of BOI effects during a SCT.

Very few studies have investigated the neural correlates of semantic richness effects in visual word recognition. Using functional magnetic resonance imaging (fMRI), Pexman and colleagues found that words with a greater number of associates (NoA; Nelson et al., 1998) showed less cortical activation than words with fewer associates in an SCT (Pexman et al., 2007). The authors attributed this to the relative efficiency of processing, with low NoA words requiring more processing time, and recruiting more cortical areas, than high NoA words. Though studied by separate labs and in separate tasks (LDT and SCT, respectively) both NoA (Müller et al., 2010) and the number of semantic features (NoF: McRae et al., 2005; Amsel, 2011) displayed unique time courses and cortical topographies relative to other lexical predictors in electroencephalography (EEG) studies. Although it is difficult to extend these findings to the present study in order to make predictions about BOI, the results of these studies clearly demonstrate that semantic richness variables can influence cortical activity during visual word recognition.

Perhaps more useful for forming predictions about BOI effects are the results of numerous studies which have revealed contributions of modality-specific areas of the brain when participants are engaged in off-line processes such as reading (Pulvermüller, 2010; Kiefer and Pulvermüller, 2012). One example is a study by Pulvermüller et al. (2005; see also Hauk and Pulvermüller, 2004). Participants were asked to make lexical decisions about action words that involved face, arm, and leg actions. They used sub-threshold TMS to stimulate areas of the motor cortex, targeting arm and leg areas of the left language-dominant hemisphere. Participants were instructed to respond in the LDT by making brief lip movements in order to avoid confounding TMS stimulation with a manual response. Pulvermüller and colleagues found that targeted TMS stimulation improved the recognition of action words in the LDT. Moreover, this effect was somatotopically mapped, so that, for example, stimulation of arm-areas enhanced processing of arm-related action words compared to leg-related action words. In related work, Desai et al. (2010) used fMRI to examine the cortical activation associated with auditory processing of sentences describing motor actions of the hand/arm (compared to sentences describing visual events or abstract behaviors). Results showed greater activation for these motor action sentences in several sensorimotor regions, including left inferior post-central sulcus and supramarginal gyrus (BA 40). If the BOI variable does indeed measure sensorimotor experience then some of these areas may also be associated with processing of high BOI words in the present study.

\section{METHOD \\ PARTICIPANTS}

The participants were 16 healthy adults, including eight men $(M=26.50$ years, $\mathrm{SD}=7.15$ years $)$ and eight women $(M=$ 22.12 years, $\mathrm{SD}=1.72$ years), all paid for participation. All participants were right-handed, monolingual English speakers, with normal or corrected-to-normal vision. Participants had no history of psychological or developmental disorders, neurological impairments, or any prescription drug use at the time of participation.

\section{MATERIALS}

\section{SELECTION OF STIMULI}

Stimuli were selected from items in the BOI rating norms acquired by Tillotson et al. (2008). A total of 72 words were selected from the norms, with 36 words rated high in BOI (e.g., belt) and 36 words rated low in BOI (e.g., sun). Care was taken to ensure that these two sets of items were matched with respect to other lexical and semantic variables that are known to influence behavior and correlated neural activity (see Table 1), and these procedures are outlined below. Following McRae and colleagues (McRae et al., 2005), a separate group of 28 participants completed an online ratings task, and were asked to list different types of features, such as physical properties (how it looks), and 
Table 1 | Mean characteristics (Standard Deviations in parentheses) for word stimuli.

\begin{tabular}{|c|c|c|c|c|c|c|c|c|c|c|}
\hline Word type & BOI & Length & NoF & Familiarity & Concreteness & Imageability & Print frequency & CD & OLD20 & PLD20 \\
\hline \multirow[t]{2}{*}{ High BOI } & 5.60 & 4.20 & 8.47 & 542.48 & 560.25 & 560.25 & 3.24 & 2.95 & 1.38 & 1.21 \\
\hline & $(0.47)$ & $(0.78)$ & $(2.44)$ & $(49.00)$ & (50.00) & $(46.00)$ & $(0.65)$ & $(0.58)$ & $(0.32)$ & $(0.26)$ \\
\hline Low BOI & $(0.59)$ & $(0.81)$ & (3.00) & (46.58) & $(48.00)$ & $(47.00)$ & $(0.80)$ & $(0.50)$ & $(0.27)$ & $(0.25)$ \\
\hline$p$-value & $<0.001$ & 0.88 & 0.61 & 0.86 & 0.55 & 0.59 & 0.90 & 0.65 & 0.87 & 0.73 \\
\hline
\end{tabular}

Note: p-values reflect difference test between high and low BOI word types; BOI = rated body-object interaction [Tillotson et al. (2008)]; Length = length in letters;

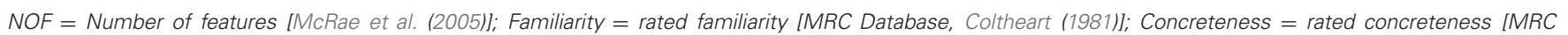

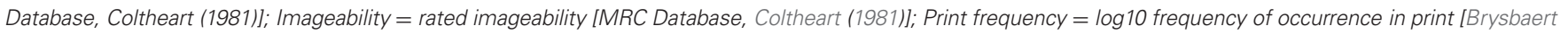

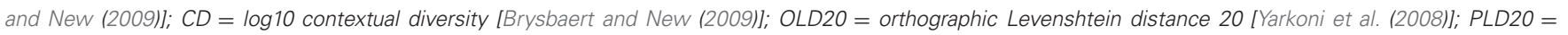
phonological Levenshtein distance 20 [Yarkoni et al. (2008)].

functional properties (what it is used for) for each target concept. For each concept, the features listed were recorded along with the number of participants who listed each feature. Results of the feature listing task showed that the high BOI and low BOI word lists did not differ on number of features per concept. In addition, the two word sets were matched for length, printed frequency, contextual diversity (Brysbaert and New, 2009), subjective familiarity (Balota et al., 2001), the mean Levenshtein distance of a word to its 20 closest orthographic and phonological neighbors (Yarkoni et al., 2008), and importantly, concreteness and imageability (Cortese and Fugett, 2004). The descriptive statistics for all the items are presented in Table 1. Finally, an additional 60 less imageable nouns (e.g., rate) were selected for the "no-go" trials, yielding a total of 132 trials. A slight imbalance in the number of critical (72) and distractor (60) trials was created in order to increase the number of critical trials included in the analysis without increasing the amount of time spent in-scanner. To be clear, the critical items were high and low BOI words for which subjectively rated imageability and concreteness had been controlled (Table 1), and thus any observed effects of BOI in this SCT can be interpreted as incremental to those of imageability or concreteness.

\section{PROCEDURE}

The study was conducted at the Seaman Family MR Research Center at the Foothills Hospital, located in Calgary, Alberta. This study was reviewed and approved by the University of Calgary Research Ethics Board. Participants were informed of any risks associated with participating, and written consent was obtained from all participants prior to partaking in the study.

A trial was initiated by a fixation marker that appeared at the center of the computer display for $1000 \mathrm{msec}$, and was then replaced by a word. Stimuli were presented for $2500 \mathrm{msec}$ with a randomized interval of $4000 \mathrm{msec} \pm 2000 \mathrm{msec}$. A variable inter-trial-interval was used to increase the detectability of the hemodynamic responses to trials (Birn et al., 2002). All stimuli were presented in a randomized order in a single block lasting $17 \mathrm{~min}$. Previous investigations of BOI effects in behavior have demonstrated a significant influence of BOI during a SCT when participants were asked to decide whether a word was easily imageable or not (Siakaluk et al., 2008b). These effects of BOI on categorization performance were quite robust, and have been observed using both manual and verbal responses during a go/no-go SCT using the imageability decision (Wellsby et al., 2011). As this was the first investigation into the brain-based correlates of BOI effects, we followed Wellsby and colleagues' procedure, adopting a go/no-go SCT using the imageability decision category. This procedure was adopted in order to facilitate the interpretation of the imaging results by using a SCT that has already demonstrated a sizable $(61 \mathrm{msec})$ behavioral effect of BOI. In addition, the imageability decision is sufficiently broad that a number of both high and low BOI items could be equally typical of the decision category, while at the same time requiring that participants engage in semantic processing in order to respond accurately. The participants' task was to decide whether each word's referent was easily imageable and to respond as quickly and as accurately as possible. Participants were instructed to respond only to the words that were easily imageable ("go" response) by pressing the button and were further instructed to refrain from making a button response to the words that were less imageable ("no-go" response). After a button press, the word would appear underlined, so as to indicate that a response had been made. Each participant first completed six practice trials, outside of the magnet room, consisting of four words that were imageable and two that were less imageable. All practice stimuli were similar in normative frequency to the experimental stimuli.

\section{ACQUISITION OF BEHAVIORAL DATA}

All stimuli were presented to participants using a rear-mounted LED projector display system (Avotec, Inc., Stuart, FL). The sequence of trials was presented using Presentation software (Neurobehavioral Systems, Albany, CA), running on a computer located outside of the magnet room. Participants' responses were recorded using a MR-compatible Lumina response pad (Cedrus Corporation, San Pedro, CA).

\section{ACOUISITION OF FMRI DATA}

Images were acquired using a 3-Tesla General Electric MR scanner, equipped with an 8-channel phased array head coil (Signa Excite; GE Healthcare, Waukesha, WI). The MR sequence for functional imaging was a single-shot gradient-recalled echo planar imaging (EPI) T2*-weighted sequence, with whole head coverage $(64 \times 64$ matrix, zero-filled to $128 \times 128$, FOV $=24 \mathrm{~cm}$, $\mathrm{TE}=30 \mathrm{msec}, \mathrm{TR}=2000 \mathrm{msec}$, flip angle $=70,31$ oblique $/$ axial 
slices, $4 \mathrm{~mm}$ thick). High-resolution T1-weighted images $(0.94 \times$ $0.94 \times 2.00 \mathrm{~mm}$ ) were collected using a $3 \mathrm{D}$ inversion recoveryprepped anatomical MRI sequence to anatomically register the functional data.

Image analyses were carried out using FEAT (FMRI Expert Analysis Tool) Version 5.98, part of FSL (FMRIB's Software Library, www.fmrib.ox.ac.uk/fsl). Images were corrected for head motion during post-processing using the intra-modal motion correction tool MCFLIRT (Jenkinson and Smith, 2001; Jenkinson et al., 2002). Prior to analysis, image data were subjected to high-pass temporal filtering (Gaussian-weighted LSF straight line fitting, with sigma $=25.0 \mathrm{~s}$ ). The following pre-statistics processing was also applied: slice-timing correction using Fourier-space time-series phase-shifting; non-brain removal using BET (Smith, 2002); spatial smoothing using a Gaussian kernel of FWHM $6 \mathrm{~mm}$; and mean-based intensity normalization of all volumes by the same factor. For all participants, time-series statistical analyses for each stimulus category (high and low BOI trials, no-go trials, and error trials) were carried out using FMRIB's Improved Linear Model (FILM) with local autocorrelation correction (Woolrich et al., 2001). Registration of high-resolution images to the MNI brain was carried out using FLIRT (Jenkinson and Smith, 2001; Jenkinson et al., 2002).

Higher-level contrasts of planned comparisons of the critical stimuli (high vs. low BOI words) across participants were carried out using FLAME (FMRIB's Local Analysis of Mixed Effects) stage 1 only (i.e., without the final MCMC-based stage) (Beckmann et al., 2003; Woolrich et al., 2004). Z-statistic images for these analyses were generated using a random effects model and a statistical threshold of $Z=2.3$, and a cluster size threshold of at least 77 contiguous voxels corresponding to a corrected $p$-value of 0.05 as determined by Monte Carlo simulations using AlphaSim (http://afni.nihm.nih.gov/afni/doc/ manual/AlphaSim). These simulations provide an estimate, for a given smoothness, of the cluster volume necessary to exceed a certain confidence in a cluster.

\section{RESULTS}

\section{BEHAVIORAL RESULTS}

Words with less than 70\% accuracy (3 high BOI and 7 low BOI, identified in the Appendix) were excluded from subsequent anal$y$ sis $^{1}$. No trials were excluded on the basis of response latency, as all responses fell between $250 \mathrm{msec}$ and $2500 \mathrm{msec}$. Responses to the high BOI words $(M=930 \mathrm{msec}, \mathrm{SD}=154 \mathrm{msec})$ were significantly faster than responses to the low BOI words $(M=$ $1083 \mathrm{msec}, \mathrm{SD}=153 \mathrm{msec}$ ), and this BOI effect was significant by subjects and by items, $t_{1}(15)=6.62, p<0.001 ; t_{2}(60)=4.66$, $p<0.001$. There was also a BOI effect in the accuracy data, as responses to the high $\mathrm{BOI}$ words $(M=98 \%, \mathrm{SD}=4 \%)$ were significantly more accurate than responses to the low BOI words $(M=93 \%, \mathrm{SD}=7 \%)$ both by subjects and by items, $t_{1}(15)=$

\footnotetext{
${ }^{1}$ The adoption of these exclusion criteria did not create a significant imbalance between high and low BOI lists in terms of the control variables reported in Table 1 (all $p$-values $>0.05$ ) and there remained a significant difference along the BOI dimension between high $(M=5.61, \mathrm{SD}=0.46)$ and low BOI $(M=$ $3.37, \mathrm{SD}=0.58)$ lists, $t(60)=16.75, p<0.001$.
}

$5.75, p<0.001 ; t_{2}(60)=3.37, p=0.001$. These findings replicate the facilitatory BOI effects reported by Bennett et al. (2011), Siakaluk et al. (2008b), and Wellsby et al. (2011).

\section{IMAGING RESULTS}

The results of the planned contrasts of high BOI and low BOI words are displayed in Figure 1 and all significant regions of activation, $z$-scores, and corresponding Talairach coordinates from these contrasts, are presented in Table 2. Several areas were significantly more active during categorization of low BOI words compared to high BOI words. These areas are part of an inhibitory control network often observed in go/no-go paradigms (e.g., Nakata et al., 2008; Simmonds et al., 2008), and comprise the right superior frontal gyrus (BA 8) including the presupplementary motor area (BA 6), the right middle frontal gyrus (BA 9), and the right inferior frontal gyrus (BA 45). In the reverse contrast, one area was significantly more active in the processing of high BOI words compared to low BOI words. That is, we observed greater activation in the left inferior parietal lobule (supramarginal gyrus, SMG, BA 40), a sensory association area involved in kinesthetic memory (Grèzes and Decety, 2001; Péran et al., 2010).

\section{DISCUSSION}

The present study was designed to investigate the neural correlates of BOI effects in semantic categorization. Analyses showed that a number of cortical areas were more active for low BOI words than for high BOI words, and our interpretation of this activity is informed by our use of the go/no-go task. That is, areas that showed more activity for low BOI words compared to high BOI words include a network thought to underlie response inhibition (Nakata et al., 2008). This relative utilization of inhibitory control mechanisms suggests that, compared to high BOI words, low BOI words don't contribute as much positive information in favor of a "go" response. Hence, more activity is observed in the inhibitory control network when participants categorize low BOI words. This is consistent with past interpretations of BOI

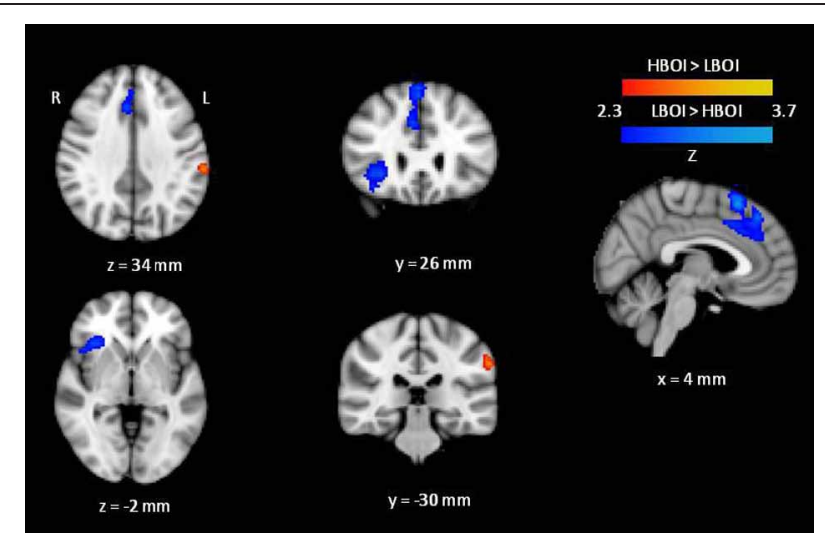

FIGURE 1 | Cortical activation maps displaying results of the high BOI (HBOI) versus low $\mathrm{BOI}$ (LBOI) contrasts. Regions showing greater activation for high $\mathrm{BOI}$ compared to low $\mathrm{BOI}$ are shown in orange. Regions showing greater activation for low $\mathrm{BO}$ compared to high $\mathrm{BO}$ are shown in Blue. 
Table 2 | Areas of significant activation in contrasts between word types.

\begin{tabular}{lllll}
\hline Contrast & Region of activation & BA & Z Score & $\begin{array}{c}\text { Talaraich coordinates } \\
\boldsymbol{x}, \boldsymbol{y}, \boldsymbol{z}\end{array}$ \\
\hline High BOI > Low BOI & Left Supramarginal Gyrus & 40 & 3.06 & $-62,-30,34$ \\
Low BOI > High BOI & Right Superior Frontal Gyrus (pre-SMA) & 6 & 3.11 & $4,22,58$ \\
& Right Superior Frontal Gyrus & 8 & 2.72 & $4,38,46$ \\
& Right Middle Frontal Gyrus & 9 & 2.42 & $48,16,26$ \\
& Right Inferior Frontal Gyrus & 45 & 2.49 & $36,28,-2$ \\
\hline
\end{tabular}

Note: Brodmann's areas (BA) should be considered estimates only.

effects on semantic categorization (Siakaluk et al., 2008b; Bennett et al., 2011), which have argued that the observation of behavioral facilitation for high BOI words stems from the relative availability of semantic information for the decision. In the present version of the go/no-go task, participants must withhold a response for abstract words. Since high BOI words are relatively richer in terms of their semantics, they contribute relatively more information to the decision than do low BOI words. In contrast, the observed cortical activation suggests that for low BOI word participants show a tendency to withhold their response, despite successfully responding "go" for these words. Again, this is consistent with the supposition that low BOI words are contributing relatively less information to the decision.

As reviewed above, the BOI effect is thought to stem from the relative availability of sensorimotor information for the go/nogo decision. We tested this assumption by contrasting areas that were significantly more active for high BOI words than low BOI words. Results of this contrast showed activation in the left inferior parietal lobule (SMG, BA 40). This area has been implicated in perception and planning of goal-oriented hand-object interactions (Russ et al., 2003; Naito and Ehrsson, 2006; Tunik et al., 2008). In addition, data from clinical populations suggests that the parietal cortex is a central area for the storage and subsequent access of motor information. Lesions to the SMG have been associated with ideomotor apraxia (Haaland et al., 2000), which is characterized by an inability to correctly plan and execute motor programs when given a verbal command. This can include the inability to correctly pantomime the use of an object (e.g., "Pantomime combing your hair.") or an inability to use objects properly in real-life situations (Wheaton and Hallett, 2007). These apraxic deficits are most commonly observed for actions that are directed toward objects or tools. Importantly, in order to qualify as ideomotor apraxia, object-knowledge and the ability to correctly recognize objects must be preserved.

During the BOI ratings task (Tillotson et al., 2008), participants were given explicit instructions to rate each word in terms of the ease with which the human body can physically interact with the word's referent, and to try to ignore other related-factors, such as how easily it can be experienced by the senses (e.g., vision, taste, etc.). As a result, the assumption is that these ratings capture the relative degree of sensorimotor experience that participants have with the object to which the word refers. It was unclear, however, to what extent these ratings actually captured differences in sensorimotor information. The present finding, that words rated high in BOI recruit areas of the brain (during an off-line visual word recognition task) that play an important role in kinesthetic memory, specifically kinesthetic memory that is involved in the correct performance of verbally cued complex actions for objects, supports the assumption that BOI ratings capture differences in sensorimotor experience.

Though the relationship between SMG activation and BOI is easily interpretable, the specificity of the observed cortical activation raises an interesting question. As reviewed in the introduction, the studies of Pulvermüller and colleagues suggest that somatotopically mapped areas of the motor cortex play a functional role in the processing of action words (Hauk and Pulvermüller, 2004; Pulvermüller et al., 2005). Indeed, a large distributed network of modality-specific areas is implicated in semantic processing (Patterson et al., 2007; Kiefer and Pulvermüller, 2012). Why, then, does a manipulation of the perceived ease of embodied interaction with a words' referent selectively recruit the SMG? Certainly, the demands of the go/no-go task could limit the contributions of other cortical areas to processing (Hargreaves et al., 2012). However, it is also worth noting that the current sets of stimuli are carefully balanced on numerous lexical and semantic dimensions (Table 1). Thus, although a distributed network of modality-specific areas may contribute to the construction of word meaning, our controlled manipulation of relative BOI may render our analysis insensitive to many of these contributions.

By manipulating BOI, we observed that the relative availability of sensorimotor information influenced activity in an area of the brain that is involved in complex motor processing. As such, our results are similar to those of other studies showing that sensorimotor and perceptual systems contribute to off-line processing such as reading (e.g., Desai et al., 2010). Studies have shown that language processing can rapidly recruit areas of the brain dedicated to perception and action, suggesting an immediate role for this information in the construction of meaning (Pulvermüller, 2010). However, there is always the possibility that this information is ancillary, and does not directly contribute to core semantic processes (Mahon and Caramazza, 2008). Just as there are many models of semantic memory, there is a great deal of variability in what researchers consider to be semantic. A broad definition of semantics as "world knowledge" would easily integrate the current findings, with BOI capturing the contribution of sensorimotor information to the processing of concepts. This broad definition has been utilized by our group to interpret the behavioral consequences of BOI in past studies (Siakaluk et al., 2008a; Bennett et al., 2011), and is also well represented 
by recent developments in cognitive neuroscience. For example, a framework like that proposed by Kiefer and Pulvermüller (2012) features concepts that are flexible, and that have representations that are distributed across numerous modality-specific informational dimensions. However, a more narrow definition of semantics that construes motor contributions as auxiliary to core semantic processing would not accommodate motor processes as constitutive of meaning, only that they interface with the conceptual system at some point during processing (Mahon and Caramazza, 2008). Note that neither side would disagree on the current data, only on the extent to which we can claim that sensorimotor information contributes to the construction of meaning.

The present results provide valuable new insight about the nature of BOI effects in visual word recognition. This study was the first test of the brain-based consequences of this variable. The results showed that higher levels of BOI are associated with activity in an area of the brain involved in kinesthetic memory,

\section{REFERENCES}

Amsel, B. (2011). Tracking real-time neural activation of conceptual knowledge using single-trial eventrelated potentials. Neuropsychologia 49, 970-983.

Balota, D., Pilotti, M., and Cortese, M. (2001). Subjective frequency estimates for 2,938 monosyllabic words. Mem. Cognit. 29, 639-647.

Barsalou, L. W. (1999). Perceptual symbol systems. Behav. Brain Sci. 22, 577-660.

Beckmann, C., Jenkinson, M., and Smith, S. M. (2003). General multilevel linear modeling for group analysis in fMRI. Neuroimage 20, 1052-1063.

Bennett, S. D. R., Burnett, A. N., Siakaluk, P. D., and Pexman, P. M. (2011). Imageability and bodyobject interaction ratings for 599 multisyllabic words. Behav. Res. Methods 43, 1100-1109.

Birn, R. M., Cox, R. W., and Bandettini, P. A. (2002). Detection versus estimation in event-related fMRI: choosing the optimal stimulus timing. Neuroimage 15, 252-264.

Brysbaert, M., and New, B. (2009). Moving beyond Kucera and Francis: a critical evaluation of current word frequency norms and the introduction of a new and improved word frequency measure for American English. Behav. Res. Methods Instrum. Comput. 41, 977-990.

Coltheart, M. (1981). MRC psycholinguistic database. Q. J. Exp. Psychol. 33A, 497-505.

Collins, A., and Loftus, E. (1975). A spreading-activation theory of semantic processing. Psychol. Rev. $82,407-428$.
Cortese, M., and Fugett, A. (2004). Imageability ratings for 3,000 monosyllabic words. Behav. Res. Methods Instrum. Comput. 36, 384-387.

Desai, R. H., Binder, J. R., Conant, L. L., and Seidenberg, M. S. (2010). Activation of sensory-motor areas in sentence comprehension. Cereb. Cortex 20, 468-478.

Dove, G. (2009). Beyond perceptual symbols: a call for representational pluralism. Cognition 110, 412-431.

Gibbs, R. W. (2006). Embodiment and Cognitive Science. New York, NY: Cambridge University Press.

Grèzes, J., and Decety, J. (2001). Functional anatomy of execution, mental simulation, observation, and verb generation of actions: a metaanalysis. Hum. Brain Mapp. 12, $1-19$.

Haaland, K., Harrington, D., and Knight, R. (2000). Neural representations of skilled movement. Brain 123, 2306-2313.

Hargreaves, I. S., White, M., Pexman, P., Pittman, D., and Goodyear, B. (2012). The question shapes the answer: the neural correlates of task differences reveal dynamic semantic processing. Brain Lang. 120, 73-78.

Hauk, O., and Pulvermüller, F. (2004). Neurophysiological distinction of action words in the fronto-central cortex. Hum. Brain Mapp. 21, 191-201. M., and Smith, S. (2002). Improved optimization for the robust and accurate linear registration and motion correction of brain images. Neuroimage 17, 825-841.

Jenkinson, M., and Smith, S. M. (2001). A global optimization method for robust affine registration of brain
Jenkinson, M., Bannister, P., Brady,

supporting the assumption of Siakaluk and colleagues that variability along the BOI dimension captures variability in the availability of sensorimotor information (2008b). Our results build upon a literature documenting effects of sensorimotor experience in reading (Wilson, 2002; Siakaluk et al., 2008a), a form of off-line processing that is somewhat removed from physical interaction with the environment and was originally conceived as the systematic manipulation of abstract symbols that are fundamentally amodal in character (Pylyshyn, 1984). With additional studies, researchers may better understand whether the contribution of sensorimotor information is indicative of a dynamic semantic system that utilizes multiple, modality-specific sources (Kiefer and Pulvermüller, 2012), or is best conceived as the manipulation of abstract structures, that interface with modality-specific processes downstream (Mahon and Caramazza, 2008). Regardless of which view one subscribes to, the present results serve to clarify and reinforce the contributions of sensorimotor experience to lexical-semantic processing.

images. Med. Image Anal. 5, 143-156.

Juhasz, B., Yap, M., Dicke, J., Taylor, S., and Gullick, M. (2011). Tangible words are recognized faster: the grounding of meaning in sensory and perceptual systems. Q. J. Exp. Psychol. 64, 1683-1691.

Keuleers, E., Lacey, P., Rastle, K., and Brysbaert, M. (2012). The British Lexicon Project: Lexical decision data for 28,730 monosyllabic and disyllabic English words. Behav. Res. Methods [Epub ahead of Print].

Kiefer, M., and Pulvermüller, F. (2012). Conceptual representations in mind and brain: theoretical developments, current evidence and future directions. Cortex [Epub ahead of Print]

Mahon, B., and Caramazza, A. (2008) A critical look at the embodied cognition hypothesis and a new proposal for grounding conceptual content. J. Physiol. Paris 102, 59-70.

McRae, K., Cree, G. S., Seidenberg, M. S., and McNorgan, C. (2005). Semantic feature production norms for a large set of living and nonliving things. Behav. Res. Methods 37, 547-559.

Morton, J. (1969). Interaction of information in word recognition. Psychol. Rev. 76, 165-178.

Müller, O., Duñabeitia, J., and Carreiras, M. (2010). Orthographic and associative neighbourhood density effects: what is shared, what is different? Psychophysiology 47, 455-466.

Myung, J., Blumstein, S., and Sedivy, J. (2006). Playing on the typewriter, typing on the piano: manipulation knowledge of objects. Cognition 98, 223-243.

Naito, E., and Ehrsson, H. H. (2006). Somatic sensation of hand-object interactive movement is associated with activity in the left inferior parietal cortex. J. Neurosci. 26, 3783-3790.

Nakata, H., Sakamoto, K., Ferretti, A., Gianni Perrucci, M., Del Gratta, C., Kakigi, R., and Luca Romani, G. (2008). Somato-motor inhibitory processing in humans: an eventrelated functional MRI study. Neuroimage 39, 1858-1866.

Nelson, D. L., McEvoy, C. L., and Schreiber, T. A. (1998). The University of South Florida Word Association, Rhyme, and Word Fragment Norms [Online]. Available: http://www.usf.edu/FreeAssociation/ Patterson, K., Nestor, P. J., and Rogers, T. T. (2007). Where do you know what you know? The representation of semantic knowledge in the human brain. Nat. Rev. Neurosci. 8 , 976-987.

Pexman, P. M., Hargreaves, I. S., Edwards, J. D., Henry, L. C., and Goodyear, B. G. (2007). The neural consequences of semantic richness: when more comes to mind, less activation is observed. Psychol. Sci. 18, 401-406.

Pexman, P. M., Lupker, S. J., and Hino, Y. (2002). The impact of feedback semantics in visual word recognition: number of features effects in lexical decision and naming tasks. Psychon. Bull. Rev. 9, 542-549.

Péran, P., Démonet, J. F., Cherubini, A., Carbebat, D., Caltagirone, C., and Sabatini, U. (2010). Mental representations of action: the neural correlates of the verbal and motor components. Brain Res. 1328, 89-103.

Pulvermüller, F. (2010). Brain-language research: where is the progress? Biolinguistics 4, 255-288. 
Pulvermüller, F., Hauk, O., Nikulin, V., and Ilmoniemi, R. J. (2005). Functional interaction of language and action: a TMS study. Eur. J. Neurosci. 21, 793-797.

Pylyshyn, Z. (1984). Computation and Cognition: Towards a Foundation for Cognitive Science. Cambridge, MA: MIT Press.

Russ, M. O., Mack, W., Grama, C. R., Lanfermann, H., and Knopf, M. (2003). Enactment effect in memory: evidence concerning the function of the supramarginal gyrus. Exp. Brain Res. 149, 497-504.

Siakaluk, P. D., Pexman, P. M., Aguilera, L., Owen, W. J., and Sears, C. R. (2008a). Evidence for the activation of sensorimotor information during visual word recognition: the bodyobject interaction effect. Cognition 106, 433-443.

Siakaluk, P. D., Pexman, P. M., Sears, C. R., Wilson, K., Locheed, K., and Owen, W. J. (2008b). The benefits of sensorimotor knowledge: body-object interaction facilitates semantic processing. Cogn. Sci. 32, 591-506.

Simmonds, D., Pekar, J., and Mostofsky, S. (2008). Meta-analysis of go/nogo tasks demonstrating that fMRI activation associated with response inhibition is task-dependent. Neuropsychologia 46, 224-232.

Smith, S. (2002). Fast robust automated brain extraction. Hum. Brain Mapp. 17, 143-155.

Tillotson, S. M., Siakaluk, P. D., and Pexman, P. M. (2008). Body-Object interaction ratings for 1,618 monosyllabic nouns. Behav. Res. Methods 40, 1075-1078.

Tunik, E., Lo, O. Y., and Adamovich, S. V. (2008). Transcranial magnetic stimulation to the frontal operculum and supramarginal gyrus disrupts planning of outcomebased hand-object interactions. J. Neurosci. 28, 14422-14427.

Wellsby, M., Siakaluk, P., Owen, W., and Pexman, P. (2011). Embodied semantic processing: the bodyobject interaction effect in a non-manual task. Lang. Cogn. 3, $1-14$.

Wheaton, L., and Hallett, M. (2007). Ideomotor apraxia: a review. $J$. Neurol. Sci. 260, 1-10.

Wilson, M. (2002). Six views on embodied cognition. Psychon. Bull. Rev. 9, 625-636.

Woolrich, M. W., Behrens, T. E. J., Beckmann, C. F., Jenkinson, M., and Smith, S. M. (2004). Multi-level linear modeling for fMRI group analysis using Bayesian inference. Neuroimage 21, 1732-1747.

Woolrich, M. W., Ripley, B. D., Brady, J. M., and Smith, S. M. (2001). Temporal autocorrelation in univariate linear modeling of fMRI data. Neuroimage 14, 1370-1386.

Yarkoni, T., Balota, D., and Yap, M. (2008). Moving beyond Coltheart's $\mathrm{N}$ : a new measure of orthographic similarity. Psychon. Bull. Rev. 15, 971-979.

Conflict of Interest Statement: The authors declare that the research was conducted in the absence of any commercial or financial relationships that could be construed as a potential conflict of interest.

Received: 11 January 2012; paper pending published: 24 January 2012; accepted: 05 February 2012; published online: 16 February 2012.

Citation: Hargreaves IS, Leonard GA, Pexman PM, Pittman DJ, Siakaluk PD and Goodyear BG (2012) The neural correlates of the body-object interaction effect in semantic processing. Front. Hum. Neurosci. 6:22. doi: 10.3389/ fnhum.2012.00022

Copyright (c) 2012 Hargreaves, Leonard, Pexman, Pittman, Siakaluk and Goodyear. This is an open-access article distributed under the terms of the Creative Commons Attribution Non Commercial License, which permits non-commercial use, distribution, and reproduction in other forums, provided the original authors and source are credited. 


\section{APPENDIX}

\section{HIGH BOI}

Belt, boot, cage, card, cart, child, cord, couch, drill, feet, friend*, gate, hat, hook, lake, lock, mail, man, mat, mate*, neck, priest, purse, room, seat, silk, stair, string, suit, sword, thing*, tool, toy, tube, vest, wheel.

\section{LOW BOI}

Ash, back, band, bay, birch, brain, brass, case, coast, frost, game*, gang, heart, jail, king, knight, lane, lint, loot*, lung, place*, prince, pump, roof, shop, slit, song*, spot, stripe, sun, trail, tar, trip*, war*, well*, witch.

*Items removed from analyses due to high error rates. 